\title{
Processing symbolic numbers: The example of distance and size effects
}

\author{
Attila Krajcsi ${ }^{1}$, Petia Kojouharova², Gábor Lengyel ${ }^{3}$ \\ ${ }^{1}$ Cognitive Psychology Department, Institute of Psychology, ELTE Eötvös Loránd University, \\ Budapest, Hungary \\ ${ }^{2}$ Research Centre for Natural Sciences, Institute of Cognitive Neuroscience and Psychology, \\ Budapest, Hungary
}

${ }^{3}$ Department of Cognitive Science, Central European University, Budapest, Hungary

According to the dominant view in the literature, several numerical cognition phenomena are explained coherently and parsimoniously by the Approximate Number System (ANS) model, which model supposes an evolutionarily old, simple representation behind many numerical tasks. We offer an alternative model, the Discrete Semantic System (DSS) to explain the same phenomena in symbolic numerical tasks. Our alternative model supposes that symbolic numbers are stored in a network of nodes, similar to conceptual or linguistic networks. The benefit of the DSS model is demonstrated through the example of distance and size effects of comparison task.

In the last few decades, the most prevalent model of numerical cognition supposed that an evolutionarily ancient, simple representation is the base of human number understanding. The model accounted for many phenomena described in the numerical cognition literature. Here, we present an alternative account, suggesting that symbolic number processing is supported by an architecture that is entirely different from the classic proposal and which alternative representation is more similar to mental conceptual networks or to the mental lexicon. With the example of distance and size effects in number comparison tasks we present several recently described phenomena supporting this alternative account.

Discussing these topics, the first section of this chapter summarizes the main features of the classic account and some of the relevant phenomena the model is based on. Then, the second section introduces our alternative account for the same phenomena. Finally, the third section presents recently discovered phenomena that demonstrate why symbolic distance and size effects cannot be accounted by the classic model, and how our alternative model can explain those effects. 


\section{The Approximate Number System account}

In 1967, Moyer and Landauer published a very simple experiment in Nature that despite its simplicity changed profoundly how cognitive scientists thought about mathematical abilities. Before their work, it was supposed that mathematics is a complicated subject for most people, and from a cognitive perspective numerical cognition is a high-level, human-specific, culture-dependent capability, which relies on complex mental processes. The work of Moyer and Landauer (1967) changed this view radically: they proposed that even symbolic number tasks may rely on such a simple mechanism as the simple perceptual representations described in psychophysics. In their experiment it was found that when participants compare single-digit numbers (e.g., which one is larger, 5 or 8?), the performance depends on the ratio of the two numbers, which behavioral pattern resembles the performance that the well-known Weber's law would generate. This meant that understanding even symbolic math, which had been believed to be a high-level and culturedependent cognitive process, might be supported by a very simple, evolutionarily old representation, that later was named the Approximate Number System (ANS).

In line with this discovery, many later studies demonstrated that the functioning of the ANS can be observed in young children or even in infants (e.g., Izard et al., 2009), and in non-human animals (e.g., Hauser, 2000). Also, many further works proposed pivotal role of the ANS in many phenomena, such as the interference of numerical and spatial information (Dehaene et al., 1993), approximate mathematical operations (Dehaene et al., 1999), understanding the cardinality principle (Piazza, 2010), developmental dyscalculia (Piazza et al., 2010), or math achievements (Halberda et al., 2008).

From the viewpoint of the present review, an important feature of the ANS is that while it is - as its name also reflects - imprecise, it also might be the root of the precise symbolic number processing, as demonstrated for example, in the original Moyer and Landauer (1967) work. Importantly, while the model supposes that the same kind of mechanisms support both the imprecise nonsymbolic (such as arrays of dots, series of sounds, basket of marbles, etc.) processing and the precise symbolic (such as Indo-Arabic digits, number words, Roman numbers, etc.) processing, this does not necessarily mean that there is a single ANS behind those numerical tasks. It is possible that there is a more noisy mechanism processing imprecise nonsymbolic stimuli, and another less noisy mechanism processing precise symbolic stimuli (Dehaene, 1997, 2007; and see a model that is considered as a possible implementation in Verguts \& Fias, 2004).

As a simple implementation of the ANS, one might imagine that numbers are stored on a continuum, where the representation of the numbers are noisy (see Figure 1). The noisy signal can be described as a Gaussian distribution where the mean of that distribution is the to be represented value, and the dispersion of the distribution is proportional to the value, i.e., the larger the number, the noisier its representation is. This simple implementation can account for the ratio effect observed, for example, in the number comparison task: the difficulty of the task depends on the overlap of the two numbers' noisy representation (see the more mathematical details of this explanation for example in Dehaene, 2007).

In this simple implementation of the ANS, the dispersion of the Gaussian distribution depends not only on the value to be represented (technically this is the mean of the distribution) but also on 
another parameter, the sensitivity of the system, expressed as the Weber fraction of the representation (Dehaene, 2007) (see left and right panels of Figure 1 for representations with different Weber fractions). Technically, the standard deviation of the distribution is the product of the to be represented value (the mean of the distribution) and the Weber fraction. The Weber fraction can be responsible either for the individual differences of the system which differences might explain partly, for example, later math achievements (Halberda et al., 2008), or for the different sensitivity of the ANS for processing symbolic and nonsymbolic stimuli.
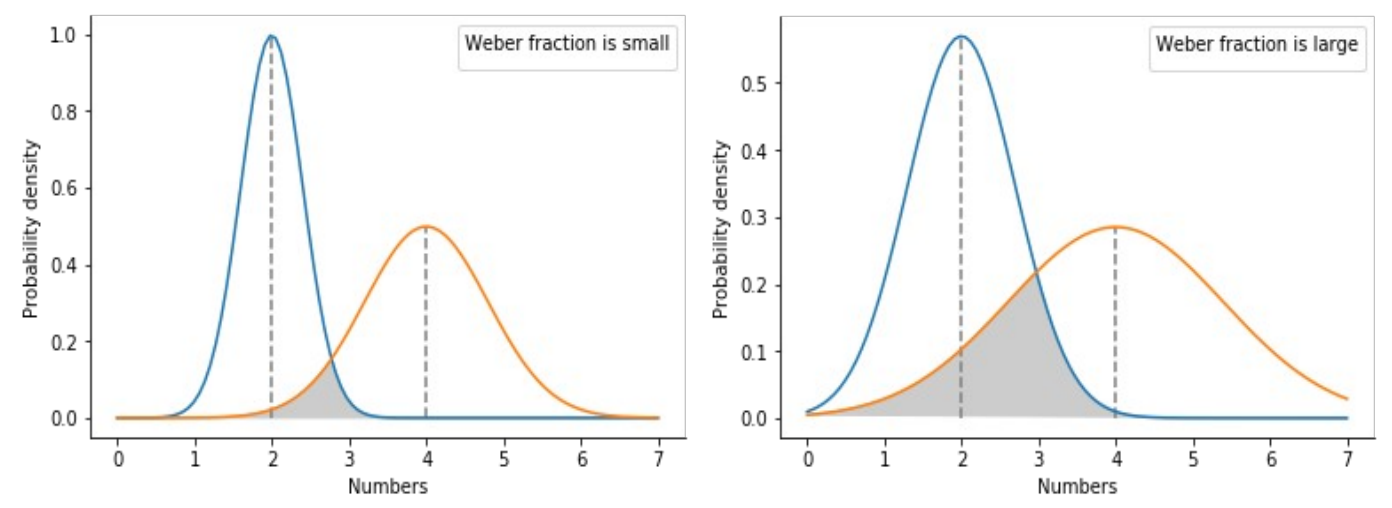

Figure 1 Possible implementation of the ANS model, displaying the representation of number 2 and number 4 with a different Weber fractions (i.e., different sensitivity; see the two panels)

In the last decades, the ANS has become the most frequently investigated representation supporting number processing and it became dominant in many aspects of numerical cognition: bases of adult number understanding, infant cognition, education, impairments of numerical abilities, or even animal cognition (Dehaene, 1992, 2007; Halberda et al., 2008; Piazza, 2010).

\section{An alternative account: Discrete Semantic System}

While the ANS model is an elegant and parsimonious solution offering explanations for a series of phenomena, in the present section we outline an alternative explanation that supposes an entirely different architecture behind the same symbolic number processing phenomena (Krajcsi et al., 2016).

Before we outline this alternative account, a technical detail should be clarified. While the psychophysical model formulates the performance of a comparison task as the ratio effect, technically, many numerical cognition works measured the distance effect (better behavioral performance with larger distance between the two numbers) or occasionally the size effect (better behavioral performance with smaller numbers). Importantly, the distance and size effects are considered as alternative approximate measurements of the ratio effect. To understand more accurately how the distance and size effects may reflect the underlying ratio effect, consider the behavioral pattern shown in the left panel of Figure 2. The figure displays the performance of a comparison task predicted by the ANS model, where the rows and the columns denote the two numbers to be compared, and the values in the cells display the difficulty of the task, darker cells denoting worse performance. The figure shows a diagonal "necktie"-like pattern, reflecting the ratio effect, which effect is based on the psychophysical model. In this context, many works measure the distance and size effects based on the regressors displayed in the right panel of Figure 2, where data 
cells with same regressor values are collapsed and performance change as a function of distance or size is measured. In the ANS model, the distance and the size effects are just two other ways to measure the ratio effect, and no matter which one someone measures, they reflect the same phenomena. The relation of the distance and size effects with the ratio effect is important not only in the ANS model, but also in our alternative explanation.
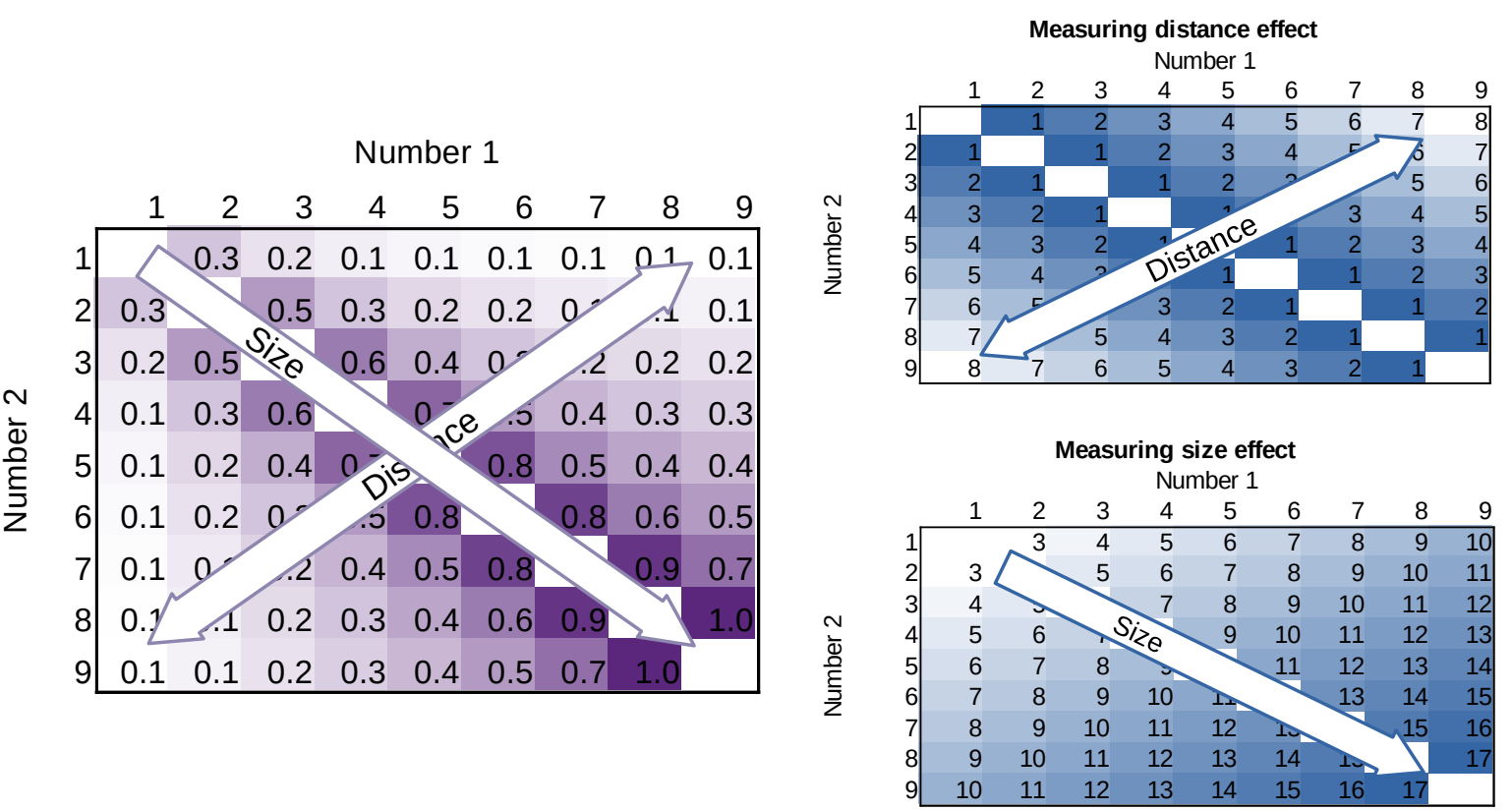

Figure 2 Left panel. The ANS model predicted performance for all combinations of numbers in a single digit comparison task. Values are measured on an arbitrary scale. More difficult number pairs are denoted by darker shades. Right panel. Distance and size effect regressors for measuring those effects in number comparison tasks.

Turning to a possible alternative explanation, it is important to highlight that whenever the distance effect can be observed in a numerical task, the literature supposes that it is the sign of the ANS. In other words, it is supposed that it is only the ANS that could generate a distance effect in a numerical task. However, this might not always be true. For example, a semantic distance-based effect was also observed in a linguistic task: in a picture naming task the size of the priming effect of the previous picture was proportional to the semantic distance between the priming and target pictures (Vigliocco et al., 2002). This semantic distance effect is conceptually and technically very similar to the numerical distance effect, because the numerical distance effect is in fact a gradual effect where the effect size depends on the semantic distance between the stimuli (i.e., on the numerical distance). Importantly, in the linguistic task most probably it is not a single (or few) continuous dimension that generates the performance pattern, like in the case of the ANS, but it might be a network of nodes (words, concepts, etc.) with specific connections. Overall, in contrast with the supposition of the current view in the numerical cognition literature, a continuous representation, such as the ANS, is not the only representation that could be the source of a numerical distance effect, but some other architectures can also produce the effect, for example a network of nodes.

Based on the idea of this potential alternative generator, our research group proposed a comprehensive alternative account for symbolic number processing that tries to account for all the 
phenomena the ANS accounts for (Krajcsi et al., 2016). Importantly, this alternative explanation deals only with symbolic numbers but not with nonsymbolic numerosities. We discuss more details of the representation supporting nonsymbolic numerosities in this alternative framework below.

In this Discrete Semantic System (DSS) model, symbolic numbers are represented by nodes and their connections (see lower part of Figure 3). (Note that the word Semantic in the name of the model refers to the fact that the model is similar to other conceptual or linguistic models accounting for semantic representations, but it does not necessarily mean that this is the system that holds the meaning of the numbers: the effects the model accounts for might be simply rooted in simple statistical properties of the numerical stimuli. See some examples of the stimulus statistics based phenomena below.) Single digits, number words, special multi-digit numbers could be nodes in that network. Connections might depend on the semantic or statistical (in the sense of environmental occurrence) properties of the nodes. For example, connection strength might depend on semantic relation (e.g., numbers with smaller distance are connected more strongly, even numbers have stronger connections with each other, etc.), while some of the semantic relations may also be based on non-mathematical properties (e.g., lucky numbers). Some other relations may depend on some statistical correlations of the stimuli (e.g., if two numbers are observed together more frequently compared to other number pairs, their relation might become stronger). This statistical property can be related to the semantic properties (numbers close to each other might be mentioned more frequently together, e.g., in a counting list), but it might also be independent of semantic properties (e.g., numbers learned by roulette or darts players based on the locations of different values on the roulette wheel or on the dartboard). The network does not necessarily include only numbers, but it might include other related concepts as well, such as “small”, “large”, “even”, “odd”, etc. (see upper part of Figure 3). (In a different conceptualization one might imagine that the whole network is only a part of the conceptual system or the mental lexicon.) This architecture is simply the architecture of various conceptual networks or mental lexicon models, applied to symbolic numbers.

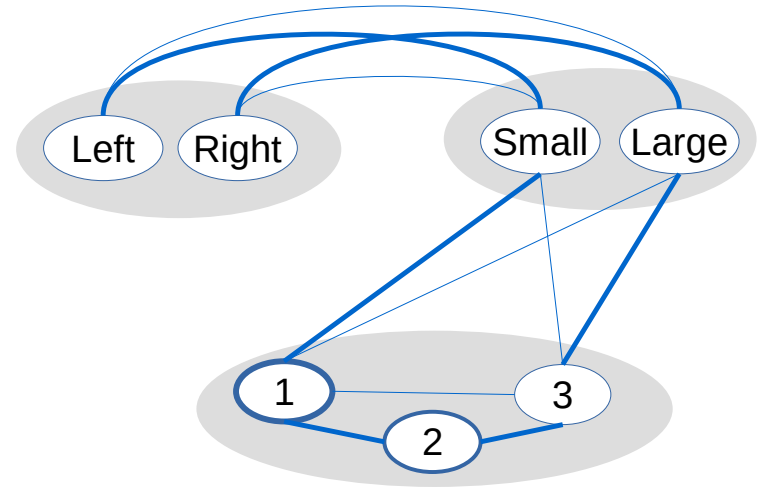

Figure 3 Schematic example of the Discrete Semantic System. Width of the lines between the nodes reflect the strength of the connections. Width of the node outlines reflect the frequency of those symbols.

We propose that this simple architecture can account for all the phenomena the ANS is supposed to account for in symbolic numbers. First, we discuss how the apparent ratio effect could be accounted for in the DSS model. In this model, comparison distance and size effects are two independent effects with two independent sources, and they form an illusionary ratio effect, which in fact has nothing to do with the psychophysical based ratio effect. In the DSS model, first, the size effect is a 
frequency effect. In everyday life, smaller numbers are more frequent than larger numbers (Dehaene \& Mehler, 1992). Both in linguistic models and in many other areas stimulus frequency heavily influences the processing speed, for example, word frequency is one of the strongest predictors of word reading time. Similarly, it is possible that smaller numbers are easier to process than larger numbers, because they are more common, leading to the size effect. Second, the distance effect might be related to the value or relative order of the numbers. According to one possibility, smaller digits might be associated with the "small" node more strongly than larger digits, and larger digits might be associated with "large” node more strongly than smaller digits (see Figure 3). In this configuration, when two numbers are compared, numbers with larger distance are easier to compare, because they have more differing associations with "large" and "small” nodes than number pairs with smaller distance. Alternatively, one might imagine that numbers with smaller numerical distance have a stronger connection than numbers with larger distance (e.g., 3 has a stronger connection with 4 than with 7; see also Figure 3). In this explanation, the distance effect could be the result of the spreading activation between the nodes: numbers with stronger connection (and with smaller distance) between them might cause more interference, leading to the distance effect. No matter which explanation could be the appropriate one, the important point is that the DSS architecture could offer a mechanism to account for the distance effect.

A last step to account for the apparent ratio effect in symbolic number comparison is to combine the size and the distance effects in the DSS model. Adding up the two effects results in a very similar pattern seen in the ANS model (see the distance and size effect components and their sum in Figure 4, and contrast this DSS description of the effect with the ANS description displayed in the left panel of Figure 2). (See more details in Krajcsi et al., 2016 about how hypothetical quantitative descriptions of the DSS could be formed.) The similarity of the DSS prediction and the ANS prediction can be captured in several ways. For example, the correlation of the cells in the two models in the one digit comparison tasks is high: the exact value depends on the exact formulation of the model predictions, and it is $r=0.89$ in the versions shown in Figures 2 and 4 . Another way to demonstrate the similarities of the two models is to contrast them as predictors of empirical behavioral performance in comparison task. In such a study, we found that it is practically impossible to find which model predicts behavioral data better, because the difference of the models is smaller than the noise in the measured data (Krajcsi et al., 2016, Experiment 1).
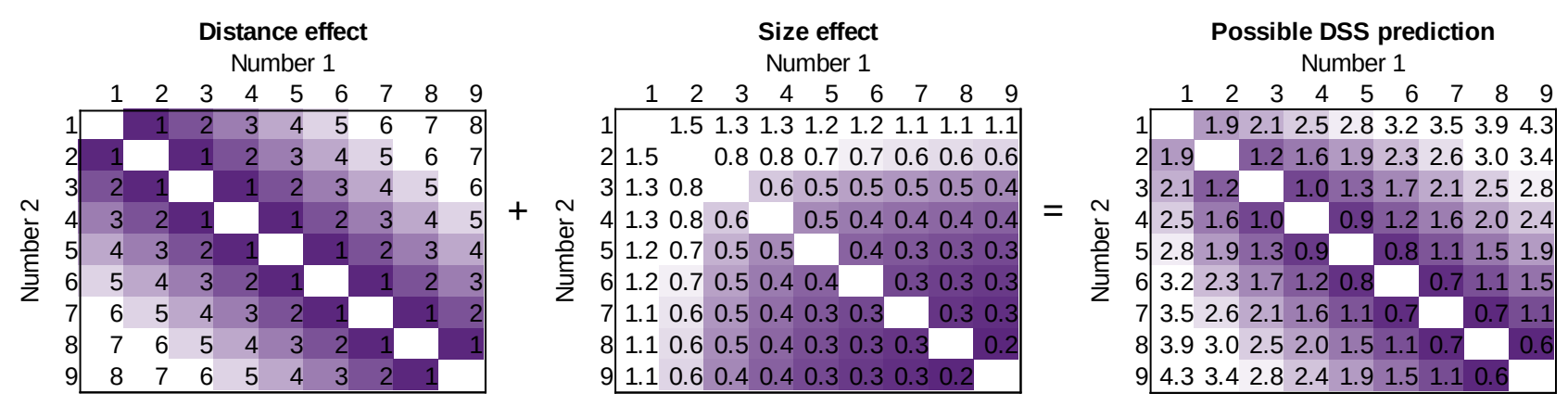

Figure 4 Predicted symbolic comparison task distance effect performance (left) and size effect performance (middle) and their sum (right) in the DSS model. Values are measured on an arbitrary scale. See more technical details of the quantitative description in Krajcsi et al. (2016). 
While in the present review we focus on the comparison distance and size effects, the DSS model can explain several other phenomena that have been attributed to the ANS model. For example, interference of the numerical and the spatial information which was originally explained by the ANS model, where spatial property of the ANS interferes with spatial representation (Dehaene et al., 1993), can also emerge in the DSS model, where "small”-"large" nodes are connected to the "left”-”right” nodes respectively (see upper part of Figure 3) (Krajcsi, Lengyel, \& Laczkó, 2018). (Note that this latter explanation is in line with other linguistic based accounts of the numerical interference effects, but those models were not intended to account for a more broader range of numerical effects.) See additional examples how the DSS may account for symbolic numerical effects in Krajcsi et al. (2016).

It was mentioned above that the DSS model accounts only for the symbolic number processing, but not the nonsymbolic numerosity processing. In this framework, we suppose that nonsymbolic stimuli are still processed by the ANS (see Figure 5 in the next section). To emphasize the difference between the classic ANS account and the alternative DSS account, while the classic view believes that both symbolic and nonsymbolic stimuli are processed by the ANS (which account we term here as the Pure ANS account), our alternative explanation supposes that it is only the nonsymbolic stimuli that are processed by the ANS, and symbolic stimuli are handled by the DSS (we term this account here as the Hybrid ANS-DSS account). Note again that the Pure ANS account supposes that while both symbolic and nonsymbolic numerical information is processed by the ANS, the account does not propose a single ANS, but two instantations of the ANS, and the two instantiations have different Weber fractions (see Figure 5).

To summarize, we propose an alternative account for symbolic number processing phenomena which phenomena formerly have been attributed to the ANS. We suggest that a simple architecture of network of nodes can account for those phenomena both qualitatively and quantitatively as demonstrated in the example of the comparison numerical distance and size effects. Overall, the DSS model offers as an appropriate prediction for the numerical effects as the ANS model.

\section{Contrasting the two accounts with new phenomena}

The previous section explained that the DSS model is as appropriate to account for symbolic number processing as the ANS model is. More generally, we found that the two models have the same predictions for almost all of the known phenomena. While this explanatory equality of the models means that the DSS model is a viable option, it also introduces a new challenge, because it is not possible to evaluate the models and tell which of them serves as a better account of those phenomena.

To overcome this issue, we designed several new tests to contrast the two models. Studies were designed to reveal phenomena for which the two models have different predictions. In this section we focus on recent results that contrast the predictions of the two models in the comparison task measuring the distance and the size effects. To summarize the main results in advance, all of our tests so far are in line only with the DSS model, revealing new details of the comparison task that cannot be reconciled with the ANS model. 
Figure 5 summarizes the two accounts to be contrasted and their main suppositions about the comparison distance and size effects in symbolic and nonsymbolic comparison tasks. While the Pure ANS account supposes that both symbolic and nonsymbolic comparisons are handled by the ANS (although with different Weber fractions), the Hybrid ANS-DSS account supposes that nonsymbolic comparison is handled by the ANS, but symbolic comparisons are processed by the DSS. Additionally, the ANS model supposes that the distance and the size effects are just two different ways to measure the ratio effect, while the DSS model proposes that the size effect depends on the frequency of the symbols and the distance effect depends on the associations of the symbols.

\section{Pure ANS account}

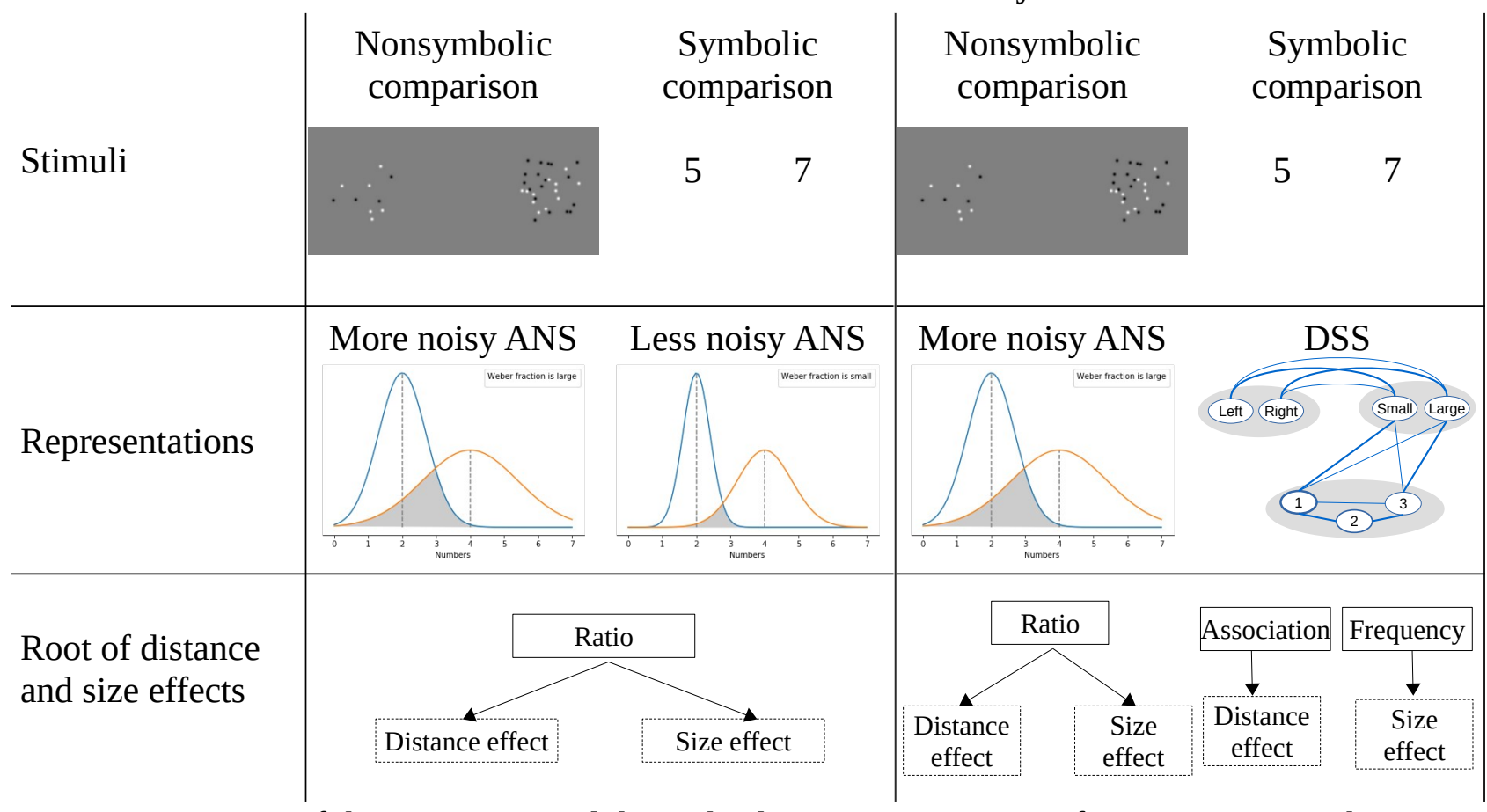

Figure 5 Summary of the Pure ANS and the Hybrid ANS-DSS accounts for comparison tasks. that in symbolic comparison task the size effect is a frequency effect (Kojouharova \& Krajcsi, 2019; Krajcsi et al., 2016) (see the first row of Table 1). In two studies, the frequency of the stimuli were manipulated, and the size effect changed accordingly. In a first study, the participants learned new symbols, and after learning their meaning, they performed comparison tasks (see the summary of the paradigm in Figure 6A). It was supposed that if the new symbols do not take the frequency information of the Indo-Arabic numbers (or other symbolic notation), which precondition proved to be true, then the size effect may reflect purely the frequency of the stimuli of the session. The results confirmed this supposition: number stimuli following everyday number frequency (Dehaene \& Mehler, 1992) caused a regular size effect, and uniform number frequency removed the size effect (Krajcsi et al., 2016). This result is in line with the DSS model, but it is in contrast with the ANS model which supposes that size effect is rooted in the ratio of the values, independent of the frequencies of the stimuli. In a next study, the same manipulation of stimulus frequency was applied to Indo-Arabic numbers. It was found that, extending the first findings, stimulus frequency also changed the size effect in Indo-Arabic numbers, although the frequency manipulation did not 
entirely change the size effect, rather the observed size effect was a combination of the previous experience with the Indo-Arabic numbers and the actual session statistics (Kojouharova \& Krajcsi, 2019).

Second, it was demonstrated that in symbolic comparison task the distance effect follows the association of the digits with the "large" and "small" properties (see the first raw of Table 1) (Kojouharova \& Krajcsi, 2018; Krajcsi \& Kojouharova, 2017). To contrast whether the distance effect is rooted in the value of the digits (prediction of the ANS) or in the association of the digits with "large" and "small” properties (prediction of the DSS model) participants compared single digit numbers, where numbers 4, 5, and 6 were omitted, creating a gap in the range (see Figure 6B). If the distance effect depends on the values of the numbers, then the distance between the two sides of the gap measured empirically should show a 3-unit distance. However, if the distance effect depends on the association of the digits and the "large"-“small” properties, then in a comparison task where all number pairs are presented uniformly, the distance between the two sides of the gap measured empirically should show a 1-unit distance (see the explanation in Figure 6B and Figure 6C). It was found both in new artificial and in Indo-Arabic notations that the distance between the two sides of the gap was 1-unit long, supporting the DSS model (Kojouharova \& Krajcsi, 2018; Krajcsi \& Kojouharova, 2017). Additionally, in the Indo-Arabic notation, the distance effect followed entirely the association statistics of the session, and it was not a combination of the current session statistic and the previous experiences as it was observed in the frequency based size effect. Again, these results confirm that the symbolic number comparison distance effect cannot be accounted for by the ANS model, because the distance effect was not driven by the values of the numbers, but by the associations between the digits and the "large”-“small” properties.

A

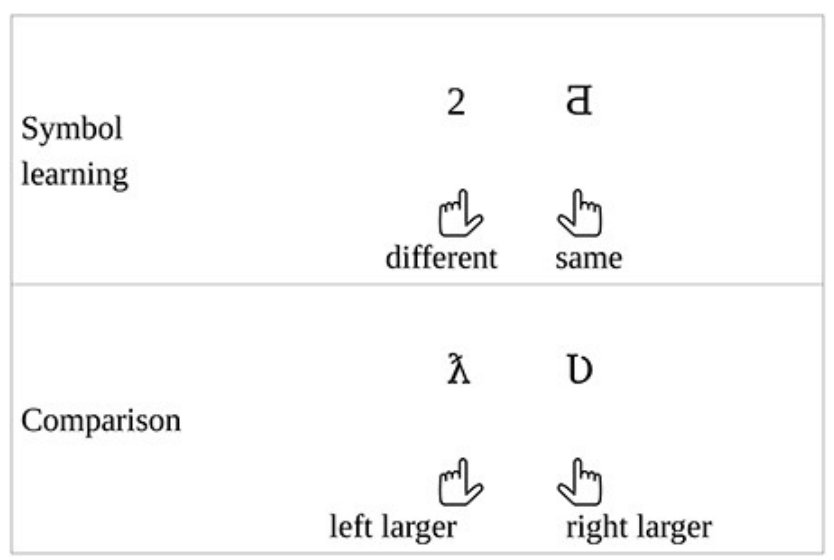

B

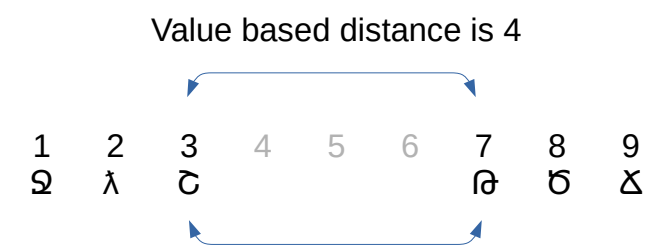

Association based distance is 1

\begin{tabular}{|c|c|c|c|c|c|c|}
\hline Example symbols & 2 & $\lambda$ & 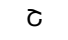 & $\theta$ & $\sigma$ & 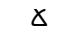 \\
\hline Meaning of the symbols & 1 & 2 & 3 & 7 & 8 & 9 \\
\hline$\%$ of being associated to "smaller" & $100 \%$ & $80 \%$ & $60 \%$ & $40 \%$ & $20 \%$ & $0 \%$ \\
\hline$\%$ of being associated to "larger" & $0 \%$ & $20 \%$ & $40 \%$ & $60 \%$ & $80 \%$ & $100 \%$ \\
\hline
\end{tabular}

Figure 6 (A) Two tasks of the new symbol comparison paradigm. After seeing a list of new symbols and their meaning in Indo-Arabic numbers, first, participants practiced the symbols in the symbol learning task, then, they compared numbers in the new symbols. (B) Numbers between 1 and 3 and 7 and 9 (i.e., a gap between 3 and 7) were used in the comparison task. If the distance effect is based on the values, then the gap should be 4-unit long. If the distance effect is based on the symbol frequency (see panel C), then the gap should be 1-unit long. (C) When numbers with a gap are presented with equal frequencies in a comparison task, the proportion of being a number "smaller" and "larger" is shown. The proportions are continuously increasing/decreasing across the gap. 
Third, in a different type of study, the psychophysical model was tested whether it can describe symbolic and nonsymbolic comparisons equally well (Krajcsi, Lengyel, \& Kojouharova, 2018). While this model has been applied to both symbolic and nonsymbolic comparison tasks, and it has repeatedly been found to be appropriate for both types of notations (e.g., Dehaene, 2007; Moyer \& Landauer, 1967), our study included more extensive tests. One of those tests investigated whether the psychophysics model describes the drift rates of the comparisons correctly in the diffusion model framework. The diffusion model supposes that in a trial evidence is accumulated until a prespecified threshold is reached (Ratcliff \& McKoon, 2008; Smith \& Ratcliff, 2004). Drift rate is the average amount of evidence that is accumulated in a time unit, and drift rate can be considered as the difficulty of the task or the efficiency of the appropriate representation which is responsible for the property the decision is based on. This model successfully explains many phenomena related to the reaction time and error rate distribution within a participant (Ratcliff \& McKoon, 2008; Smith \& Ratcliff, 2004). In the ANS model, as in similar psychophysical models, it is supposed that as the two to-be-compared values get closer (in terms of ratio), the drift rate approaches zero (Dehaene, 2007; Palmer et al., 2005). For example, in a nonsymbolic dot comparison task, as the two values become more and more similar, the participant becomes unsure which array has more items. Our analysis revealed that in the nonsymbolic comparison task the drift rate indeed approached zero as the task became more difficult. However, in the symbolic comparison task the drift rate approached a non-zero value. This latter result is in line with the common sense observation that even if comparing two symbolic numbers gets harder as the distance (or ratio) decreases, it never becomes impossible to solve (as it would be impossible for a nonsymbolic comparison task). Again, the results revealed that while nonsymbolic comparison works according to the psychophysical ANS model, symbolic comparison works differently, and the psychophysical model cannot describe it correctly (see the first row of Table 1).

Fourth, related to the previous properties and findings, we may state that in the symbolic comparison task the distance and the size effects dissociate (second row of Table 1). In the studies described above where size effects were manipulated by utilizing different frequencies of the numbers, while the size effects changed according to the stimulus frequency, the distance effect was not influenced (Kojouharova \& Krajcsi, 2019; Krajcsi et al., 2016). Similarly, in the studies where the distance effect was manipulated by presenting a number range with a gap, the size effect still depended on the frequencies of the digit (Kojouharova \& Krajcsi, 2018; Krajcsi \& Kojouharova, 2017). Thus, unlike the prediction of the ANS model, distance and size effects can be modified and manipulated independently.

Fifth, related to the independence and dissociation of the distance and the size effects described in the previous point, the two accounts have different predictions about the correlation of the slopes of the distance and size effects (third row in Table 1). In the ANS model, the slope of the distance and size effects are influenced only by the ratio effect slope which in turn is influenced only by the Weber fraction (Dehaene, 2007). (This is true even even if the slopes of the ratio, distance and size effects show some non-monotonic relation with the Weber fraction, see Chesney, 2018; and Krajcsi, 2020.) In other words, in the ANS model, if the distance and the size effects are measured independently, then actually the same construct is measured in two different ways, and the two indexes should correlate perfectly. In contrast, according to the hybrid ANS-DSS account, this perfect correlation could be expected only in nonsymbolic comparison, while symbolic comparison 
distance and size effects might be independent (see again Figure 5). In a study, measuring the correlation of the slope of the distance effect and the slope of the size effect in nonsymbolic and symbolic comparison tasks, it was found that in nonsymbolic comparison after correcting for the reliability-caused attenuation of correlation, the correlation was practically 1 , while in symbolic comparison the same correlation was not significantly different from 0 (Krajcsi, 2017). Again, this result argues for different types of mechanisms behind symbolic and nonsymbolic comparison, in line with the hybrid ANS-DSS account.

Sixth, the already mentioned flexibility of the symbolic distance and size effects are also informative in the present investigation. As described above, the symbolic distance effect proved to be highly flexible, almost entirely relying on the stimulus statistics of the session and ignoring former experience (Kojouharova \& Krajcsi, 2018), while the symbolic size effect is less flexible, combining the statistics of the actual session and former experience (Kojouharova \& Krajcsi, 2019). In the pure ANS account the two effects should show the same flexibility (and they should not be modified by those stimulus statistics in the first place), again suggesting that symbolic numbers are compared by the DSS.

\begin{tabular}{|c|c|c|c|c|}
\hline & \multicolumn{2}{|c|}{ Symbolic } & \multicolumn{2}{|c|}{ Nonsymbolic } \\
\hline & Distance effect & Size effect & Distance effect & Size effect \\
\hline Source of the effect & $\begin{array}{l}\text { Large-small } \\
\text { association of the } \\
\text { numbers } \\
\text { (Kojouharova \& } \\
\text { Krajcsi, 2018; Krajcsi } \\
\text { \& Kojouharova, 2017) }\end{array}$ & $\begin{array}{l}\text { Frequency of the } \\
\text { symbols } \\
\text { (Kojouharova \& } \\
\text { Krajcsi, 2019; } \\
\text { Krajcsi et al., } \\
\text { 2016) }\end{array}$ & \multicolumn{2}{|c|}{$\begin{array}{l}\text { Psychophysics model: Ratio } \\
\text { effect according to Weber's law } \\
\text { (Krajcsi, Lengyel, \& } \\
\text { Kojouharova, 2018) }\end{array}$} \\
\hline $\begin{array}{l}\text { Independence } \\
\text { (Kojouharova \& Krajcsi, } \\
\text { 2018, 2019; Krajcsi et } \\
\text { al., 2016; Krajcsi \& } \\
\text { Kojouharova, 2017) }\end{array}$ & \multicolumn{2}{|c|}{ Dissociation of the effects } & \multicolumn{2}{|c|}{$\begin{array}{l}\text { No dissociation of the effects is } \\
\text { observed }\end{array}$} \\
\hline $\begin{array}{l}\text { Correlation of the slopes } \\
\text { (Krajcsi, 2017) }\end{array}$ & \multicolumn{2}{|l|}{ Independent } & \multicolumn{2}{|l|}{ Strongly correlate } \\
\hline $\begin{array}{l}\text { Flexibility for the } \\
\text { statistics of the stimuli }\end{array}$ & $\begin{array}{l}\text { Highly flexible } \\
\text { (Kojouharova \& } \\
\text { Krajcsi, 2018) }\end{array}$ & $\begin{array}{l}\text { Moderately } \\
\text { flexible } \\
\text { (Kojouharova \& } \\
\text { Krajcsi, 2019) }\end{array}$ & Rigid? & \\
\hline
\end{tabular}

Table 1 Properties of the distance and size effects in symbolic and nonsymbolic comparison tasks. Flexibility of the nonsymbolic effects has not been tested yet, as noted by the question mark. 
Overall, these results show that (a) the properties of the symbolic comparison and the nonsymbolic comparison differ, and (b) while the properties of the distance and the size effects are similar within a nonsymbolic comparison task, the properties of the two effects are different within a symbolic comparison task (Table 1). Both of those findings are in line only with the hybrid ANS-DSS account but not with the pure ANS account.

\section{Conclusions}

In the last decades, the ANS model has been the dominant account in explaining many phenomena related to numerical understanding. Here, we briefly described an alternative model (DSS) with an entirely different architecture, which model is also capable of explaining the symbolic phenomena that has been attributed to the ANS. The current review summarized several recently described effects of number comparison that cannot be explained by the classic pure ANS account, but only by our alternative hybrid ANS-DSS account.

This alternative account is in line with several similar alternative models (e.g., Leth-Steensen et al., 2011; Pinhas \& Tzelgov, 2012; Proctor \& Cho, 2006; Tzelgov et al., 2009; Verguts et al., 2005; Verguts \& Van Opstal, 2014), although the scope of these similar models is less comprehensive than the intended scope of the DSS model. Also, the present model extends and specifies the ideas of many recent works suggesting that symbolic and nonsymbolic numerical information is processed differently (e.g., Leibovich \& Ansari, 2016; Lyons et al., 2015; Noël \& Rousselle, 2011; Sasanguie et al., 2014; Schneider et al., 2017). Our proposal together with the increasing number of publications discussing the limitations of the ANS model and offering solutions for some of the issues can form a new framework of numerical cognition, which framework can integrate the results of the recent years more successfully than the current dominant view of number processing.

\section{Acknowledgments}

The work of Attila Krajcsi was supported by the National Research, Development and Innovation Fund (NKFI 132165), CELSA Research Fund (CELSA/19/011) and ELTE Faculty of Education and Psychology.

\section{References}

Chesney, D. (2018). Numerical distance effect size is a poor metric of approximate number system acuity. Attention, Perception, \& Psychophysics, 80(5), 1057-1063. https://doi.org/10.3758/s13414-018-1515-X

Dehaene, S. (1992). Varieties of numerical abilities. Cognition, 44, 1-42. https://doi.org/10.1016/0010-0277(92)90049-N

Dehaene, S. (1997). The number sense: How the mind creates mathematics. Oxford University Press. 
Dehaene, S. (2007). Symbols and quantities in parietal cortex: Elements of a mathematical theory of number representation and manipulation. In P. Haggard, Y. Rossetti, \& M. Kawato (Eds.), Sensorimotor foundations of higher cognition: Vol. XXII (pp. 527-574). Harvard University Press.

Dehaene, S., Bossini, S., \& Giraux, P. (1993). The mental representation of parity and mental number magnitude. Journal of Experimental Psychology: General, 122, 371-396. https://doi.org/10.1037/0096-3445.122.3.371

Dehaene, S., \& Mehler, J. (1992). Cross-linguistic regularities in the frequency of number words. Cognition, 43, 1-29. https://doi.org/10.1016/0010-0277(92)90030-L

Dehaene, S., Spelke, E. S., Pinel, P., Stanescu, R., \& Tsivkin, S. (1999). Sources of Mathematical Thinking: Behavioral and Brain-Imaging Evidence. Science, 284(5416), 970-974.

Halberda, J., Mazzocco, M. M. M., \& Feigenson, L. (2008). Individual differences in non-verbal number acuity correlate with maths achievement. Nature, 455(7213), 665-668. https://doi.org/10.1038/nature07246

Hauser, M. D. (2000). Wild minds: What animals really think. Henry Holt.

Izard, V., Sann, C., Spelke, E. S., \& Streri, A. (2009). Newborn infants perceive abstract numbers. Proceedings of the National Academy of Sciences, 106(25), 10382-10385. https://doi.org/10.1073/pnas.0812142106

Kojouharova, P., \& Krajcsi, A. (2018). The Indo-Arabic distance effect originates in the response statistics of the task. Psychological Research. https://doi.org/10.1007/s00426-018-1052-1

Kojouharova, P., \& Krajcsi, A. (2019). Two components of the Indo-Arabic numerical size effect. Acta Psychologica, 192, 163-171. https://doi.org/10.1016/j.actpsy.2018.11.009

Krajcsi, A. (2017). Numerical distance and size effects dissociate in Indo-Arabic number comparison. Psychonomic Bulletin \& Review, 24(8), 927-934. https://doi.org/10.3758/s13423-016-1175-6

Krajcsi, A. (2020). Ratio effect slope can sometimes be an appropriate metric of the approximate number system sensitivity. Attention, Perception, \& Psychophysics. https://doi.org/10.3758/s13414-019-01939-6 
Krajcsi, A., \& Kojouharova, P. (2017). Symbolic Numerical Distance Effect Does Not Reflect the Difference between Numbers. Frontiers in Psychology, 8. https://doi.org/10.3389/fpsyg.2017.02013

Krajcsi, A., Lengyel, G., \& Kojouharova, P. (2016). The Source of the Symbolic Numerical Distance and Size Effects. Frontiers in Psychology, 7. https://doi.org/10.3389/fpsyg.2016.01795

Krajcsi, A., Lengyel, G., \& Kojouharova, P. (2018). Symbolic number comparison is not processed by the analogue number system: Different symbolic and nonsymbolic numerical distance and size effects. Frontiers in Psychology, 9. https://doi.org/10.3389/fpsyg.2018.00124

Krajcsi, A., Lengyel, G., \& Laczkó, Á. (2018). Interference between number magnitude and parity: Discrete representation in number processing. Experimental Psychology, 65(2), 71-83. https://doi.org/10.1027/1618-3169/a000394

Leibovich, T., \& Ansari, D. (2016). The symbol-grounding problem in numerical cognition: A review of theory, evidence, and outstanding questions. Canadian Journal of Experimental Psychology/Revue Canadienne de Psychologie Expérimentale, 70(1), 12-23. https://doi.org/ 10.1037/сер0000070

Leth-Steensen, C., Lucas, J., \& Petrusic, W. M. (2011). Modelling SNARC by using polarity codes to adjust drift rates. Proceedings of the 27th Annual Meeting of the International Society for Psychophysics, Herzliya, Israel, 357-362.

Lyons, I. M., Ansari, D., \& Beilock, S. L. (2015). Qualitatively different coding of symbolic and nonsymbolic numbers in the human brain. Human Brain Mapping, 36(2), 475-488. https://doi.org/10.1002/hbm.22641

Moyer, R. S., \& Landauer, T. K. (1967). Time required for Judgements of Numerical Inequality. Nature, 215(5109), 1519-1520. https://doi.org/10.1038/2151519a0

Noël, M.-P., \& Rousselle, L. (2011). Developmental Changes in the Profiles of Dyscalculia: An Explanation Based on a Double Exact-and-Approximate Number Representation Model. Frontiers in Human Neuroscience, 5. https://doi.org/10.3389/fnhum.2011.00165

Palmer, J., Huk, A. C., \& Shadlen, M. N. (2005). The effect of stimulus strength on the speed and accuracy of a perceptual decision. Journal of Vision, 5(5), 1-1. https://doi.org/10.1167/5.5.1 
PREPRINT Krajcsi, Kojouharova, Lengyel: Processing symbolic number comparison task 15/16

Piazza, M. (2010). Neurocognitive start-up tools for symbolic number representations. Trends in Cognitive Sciences, 14(12), 542-551. https://doi.org/10.1016/j.tics.2010.09.008

Piazza, M., Facoetti, A., Trussardi, A. N., Berteletti, I., Conte, S., Lucangeli, D., Dehaene, S., \& Zorzi, M. (2010). Developmental trajectory of number acuity reveals a severe impairment in developmental dyscalculia. Cognition, 116(1), 33-41.

https://doi.org/10.1016/j.cognition.2010.03.012

Pinhas, M., \& Tzelgov, J. (2012). Expanding on the Mental Number Line: Zero Is Perceived as the “Smallest.” Journal of Experimental Psychology: Learning, Memory, and Cognition, 38(5), 1187-1205. https://doi.org/10.1037/a0027390

Proctor, R. W., \& Cho, Y. S. (2006). Polarity correspondence: A general principle for performance of speeded binary classification tasks. Psychological Bulletin, 132(3), 416-442. https://doi.org/10.1037/0033-2909.132.3.416

Ratcliff, R., \& McKoon, G. (2008). The Diffusion Decision Model: Theory and Data for TwoChoice Decision Tasks. Neural Computation, 20(4), 873-922. https://doi.org/10.1162/neco.2008.12-06-420

Sasanguie, D., Defever, E., Maertens, B., \& Reynvoet, B. (2014). The approximate number system is not predictive for symbolic number processing in kindergarteners. The Quarterly Journal of Experimental Psychology, 67(2), 271-280. https://doi.org/10.1080/17470218.2013.803581

Schneider, M., Beeres, K., Coban, L., Merz, S., Susan Schmidt, S., Stricker, J., \& De Smedt, B. (2017). Associations of non-symbolic and symbolic numerical magnitude processing with mathematical competence: A meta-analysis. Developmental Science, 20(3). https://doi.org/10.1111/desc.12372

Smith, P. L., \& Ratcliff, R. (2004). Psychology and neurobiology of simple decisions. Trends in Neurosciences, 27(3), 161-168. https://doi.org/10.1016/j.tins.2004.01.006

Tzelgov, J., Ganor-Stern, D., \& Maymon-Schreiber, K. (2009). The representation of negative numbers: Exploring the effects of mode of processing and notation. Quarterly Journal of Experimental Psychology, 62(3), 605-624. https://doi.org/10.1080/17470210802034751 
Verguts, T., \& Fias, W. (2004). Representation of Number in Animals and Humans: A Neural Model. Journal of Cognitive Neuroscience, 16(9), 1493-1504. https://doi.org/10.1162/0898929042568497

Verguts, T., Fias, W., \& Stevens, M. (2005). A model of exact small-number representation. Psychonomic Bulletin \& Review, 12(1), 66-80. https://doi.org/10.3758/BF03196349 Verguts, T., \& Van Opstal, F. (2014). A Delta-Rule Model of Numerical and Non-Numerical Order Processing. Journal of Experimental Psychology: Human Perception and Performance, 40(3), 1092-1012. https://doi.org/10.1037/a0035114

Vigliocco, G., Vinson, D. P., Damian, M. F., \& Levelt, W. (2002). Semantic distance effects on object and action naming. Cognition, 85(3), B61-B69. https://doi.org/10.1016/S00100277(02)00107-5 